\title{
Claroscuros en el desarrollo del Estado de México. Un análisis espacial del Índice de Desarrollo Humano
}

\section{Jorge luts Moranchel-Bustos* y Yolanda Carbajal Suarez**}

\section{RESUMEN}

Se analiza la forma en que se distribuye el Índice de Desarrollo Humano (IDH) y sus componentes en los 125 municipios del Estado de México. El objetivo es determinar si estos fenómenos se encuentran en el territorio de forma dispersa o tienen algún grado de concentración. A partir del uso de técnicas propias del análisis exploratorio de datos espaciales, se confirma la presencia de heterogeneidad y dependencia espacial positiva en el IDH, además de sus componentes sobre nivel de educación e ingresos, no así para el nivel de salud.

Palabras clave: Estado de México, desarrollo humano, efectos espaciales, heterogeneidad espacial, dependencia espacial.

Clasificación JEL: O15

* Estudiante del Doctorado en Ciencias Económico Administrativas, Universidad Autónoma del Estado de México, Estado de México, México. Correo electrónico: jlmoranchel@gmail.com.

** Profesora-investigadora, Facultad de Economía, Universidad Autónoma del Estado de México, Estado de México, México. Correo electrónico: ycarbajals@uaemex.mx. 


\section{ABSTRACT \\ Contrasts in the development of the State of Mexico. A spatial analysis of the Human Development Index}

This paper analyzes the way in which the Human Development Index and its component were distributed in 125 municipalities of the Estado de México. The objective is to determine if these phenomena are in the territory in a dispersed way or have some degree of concentration. Using the techniques of exploratory analysis of spatial data showed heterogeneity and positive spatial dependence to HDI, education and income components, but health component scattered or differentially concentrated around the State.

Keywords: State of Mexico, human development, spatial effects, spatial heterogeneity, spatial dependence.

\section{JEL Classification: $\mathrm{O} 15$}

\section{INTRODUCCIÓN}

Durante décadas ha existido en el debate teórico la preocupación de explicar las razones por las que los países y sus regiones presentan importantes heterogeneidades no solo con otros territorios sino al interior de una zona, lo que, sin duda, redunda en la presencia de disparidades significativas en el nivel de vida de la población. Al respecto, los pioneros del desarrollo sostienen la hipótesis de que los territorios son subdesarrollados pues no han logrado establecer un crecimiento económico sostenido, lo que, en consecuencia, imposibilita aumentar el ingreso de su población (García-Docampo, 2007; Meier y Stiglitz, 2002; Carrillo-Huerta, 2001).

Todaro (1988), con un enfoque más amplio, destaca la importancia de las variables económicas; sin embargo, considera que el crecimiento de esta naturaleza resulta un componente más, pues el desarrollo implica un proceso multidimensional que requiere de la reorganización y reorientación de los sistemas económicos y sociales, donde no basta con elevar los niveles de renta o producción, en tanto se deben generar transformaciones significativas de las estructuras institucionales, sociales y administrativas; además, plantea que el progreso debe modificar actitudes de la gente e, incluso, costumbres y creencias. 
Así, la correlación única entre crecimiento y desarrollo se ha rebasado: porque el desarrollo debe entenderse como el estado donde la sociedad ha obtenido los medios necesarios para vivir mejor (Gago, 1993; Todaro, 1982). Los teóricos lo consideran producto de un aumento en los ingresos, factor que no debe asumirse como la variable endógena final, en tanto también influyen aquellos indicadores que miden la calidad y nivel de vida de la población (Acemoglu, 2009). Así, el término desarrollo adquiere un carácter multidisciplinario y multidimensional (Becerra-Lois y Pino-Alonso, 2005; Todaro, 1988); suma, además, la reducción o eliminación de la pobreza, el desempleo y la desigualdad; más aún, indicadores sociales no económicos como salud, sanidad, escolaridad, alfabetización y vivienda.

En ese sentido, Sen (2001b) explica que el desarrollo se consigue mediante el fortalecimiento de las capacidades de las personas, visión formalizada dentro del IDH del Programa de Naciones Unidas para el Desarrollo (PNUD), cuyo objetivo es medir el conjunto de capacidades y libertades de los individuos para elegir entre formas de vida alternativas. Este índice es importante porque considera tres de los principales indicadores del bienestar de las sociedades: salud -posibilidad de gozar de una vida larga y sana-, educación -capacidad de adquirir conocimientos- e ingreso -oportunidad de tener recursos que permitan un nivel de vida digno- (PNUD, 2014).

Así, el análisis del desarrollo representa una de las principales preocupaciones actuales, centrado en el debate de diversas teorías económicas contemporáneas (Mozas-Moral y Bernal-Jurado, 2006; Becerra-Lois y Pino-Alonso, 2005) tendientes a explicar desde diferentes perspectivas por qué existen territorios que presentan atrasos, con claras divergencias entre el crecimiento económico e indicadores de bienestar como el IDH.

Esta problemática se presenta de forma evidente en muchas entidades federativas del país, como el Estado de México: pese a su importancia por los significativos niveles de industrialización y una fuerte vinculación con el mercado externo -que aporta $8.7 \%$ al producto interno bruto total nacional y $7.25 \%$ al manufacturero (INEGI, 2017)-, se ubica en el décimo sexto lugar nacional por su IDH de 0.745 (PNUD, 2014), apenas por arriba del umbral del desarrollo humano medio. Asimismo, implica disparidades considerables en el nivel de desarrollo de los 125 municipios que lo integran: por un lado existen aquéllos, 
predominantemente urbanos, donde existe gran concentración poblacional, con alta participación de actividades industriales y de servicios, así como presencia superior de capital y tecnología, lo cual propicia mayores niveles de desarrollo; por otro, se encuentran los que cuentan con elevados niveles de pobreza y marginación, con escasa densidad poblacional, donde prevalecen las actividades del sector primario, con escasa o nula productividad y tecnología, caracterizados por bajos niveles de progreso.

Tal desigualdad territorial repercute en el nivel de adelanto de los diferentes municipios del Estado de México, uno de los mayores retos por afrontar. Este trabajo, además de ayudar a entender su realidad a nivel municipal y definir posibles líneas de investigación, busca abonar a los escasos estudios existentes en torno al desarrollo de estados mexicanos.

Esta investigación no busca estudiar la validez ni las variables que se consideran para la construcción del IDH, solo lo emplea para un acercamiento exploratorio encaminado al descubrimiento de patrones de comportamiento en los datos y el establecimiento de hipótesis de asociación espacial, a partir del uso de herramientas gráficas y descriptivas.

El objetivo de este trabajo es analizar la forma en que se distribuye el IDH y sus componentes en los 125 municipios del Estado de México, abordados de forma individual, con la finalidad de determinar si estos fenómenos se encuentran en el territorio de forma dispersa o tienen algún grado de concentración espacial, mediante el Análisis Exploratorio de Datos Espaciales (AEDE) (Chasco-Yrigoyen, 2003b; Anselin y Bera, 1998); al mismo tiempo, se busca determinar la existencia de autocorrelación espacial entre ellos a partir del índice local de Moran y los indicadores locales de asociación espacial ${ }^{1}$ (Anselin, 1995). Esta herramienta resulta trascendental para acercarse a la interpretación de los hechos socioeconómicos producidos en el espacio geográfico, pues permite encontrar patrones de comportamientos y anomalías en la estructura de los datos, y con ello tener una visión general de la localización de las variables, destinada a la realización de futuros estudios sobre las causas de estos fenómenos.

Local Indicator of Spatial Asociation (LISA). 
Para ello, el trabajo está dividido en tres apartados, además de la introducción y las conclusiones. En el primero se abordan algunos aspectos teóricos del desarrollo; en el segundo se establece la metodología a partir del análisis exploratorio de datos espaciales; y, finalmente, en el tercero se discuten los resultados obtenidos.

\section{Del desarRollo al DeSARROLlo humano}

Al hablar del desarrollo se asume que representa un progreso, un proceso de transición hacia una condición de satisfacción socioeconómica de bienestar, que otorga a los habitantes de un territorio específico oportunidades equitativas y homogéneas (Sen, 2001a); no conseguirlo evidencia un acceso desigual a dichas ventajas, lo que repercute de forma directa en las capacidades de la población (Sen, 2001b; 1996) y, por tanto, limita sus posibilidades de desarrollo humano.

Sen (1996) sostiene que el enfoque de las capacidades se sustenta en la tesis de que el nivel de desarrollo de un territorio y el bienestar de sus habitantes debe centrarse en ampliar las posibilidades reales de los individuos para elegir el tipo de vida que consideran valioso. En ese sentido, las capacidades implican las posibles combinaciones de funcionamientos (ser y hacer) que una persona puede lograr, tan elementales como estar bien nutrido y libre de enfermedades, ser educado y contar con un buen empleo.

El concepto de desarrollo humano nace influido por el enfoque de capacidades y los trabajos de Sen (2001a, 1990), una propuesta teórica que propone estudiar y evaluar el bienestar y la calidad de vida de las personas de forma distinta al enfoque económico (Sen, 2001a; 1996). En consecuencia, el PNUD (1990) lo define como un proceso en el cual se amplían las oportunidades del ser humano y que conlleva rasgost esenciales como disfrutar de una vida prolongada y saludable (nivel de salud), adquirir conocimientos (nivel de educación) y acceder a los recursos necesarios para lograr un grado de vida decente (nivel de ingresos); a partir de las capacidades citadas integra el IDH considerando la esperanza de vida al nacer (mortalidad infantil), el promedio de año de escolarización (presente y futura) y el ingreso, respectivamente. Para su construcción se utilizan datos socioeconómicos georreferenciados, lo que permite analizarlo desde la óptica del AEDE. A partir de 1990 este índice se desarrolló para países; desde 2003, cada dos años, 
para entidades federativas de México, y cada cinco, para cada uno de los municipios del país (PNUD, 2014).

\section{El ANÁlisis EXPLORATORIO de DATOS ESPACIALES}

El AEDE es una subdisciplina perteneciente al denominado Análisis Exploratorio de Datos, la cual representa un campo muy novedoso e interesante para el análisis económico y de las ciencias sociales (Chasco-Yrigoyen, 2003b), sobre todo en el desarrollo metodológico cuantitativo actual (Buzai y Baxendale, 2009); incorpora al análisis estadístico la ubicación y distribución de los fenómenos de estudio de forma específica dentro de un territorio, lo que permite establecer de mejor forma las interacciones existentes entre las variables analizadas (Haining, 1993).

Tanto Anselin y Bera (1998), como Chasco-Yrigoyen (2009), definen al AEDE como "el conjunto de técnicas que describen y visualizan las distribuciones espaciales, identifican localizaciones atípicas o 'atípicos espaciales' (spatial outliers), descubren esquemas de asociación espacial, agrupamientos (clusters) o puntos calientes (hot spots), los cuales sugieren estructuras espaciales u otras formas de heterogeneidad espacial".

La naturaleza georreferenciada de los datos permite visualizar las relaciones socioespaciales que se ejercen multidimensionalmente en un territorio (Moreno-Serrano y Vayá-Valcarse, 2000), además de descubrir patrones, relaciones potenciales e hipótesis que pueden no ser consideradas por las teorías; en consecuencia, esta metodología cobra relevancia y pertinencia científica.

Los métodos usados por el AEDE son de carácter descriptivo, pues brinda información estructural del comportamiento de variables y permite la observación de casos anómalos o outliers (Buzai y Baxendale, 2009; Chasco- Yrigoyen, 2003b). La detección de estructuras espaciales en las variables geográficas permite la formulación de hipótesis previas para la modelización econométrica y contrastar la significancia de las relaciones observadas (Chasco-Yrigoyen, 2003a): es la puerta de entrada a la econometría espacial.

Para la identificación de tendencias espaciales y la detección de datos anómalos se realiza el análisis de dos grandes efectos espaciales: 
la heterogeneidad y la dependencia espacial (Chasco- Yrigoyen, 2003a; Moreno-Serrano y Vayá-Valcarse, 2000; Anselin y Bera, 1998).

La heterogeneidad espacial surge cuando existen datos de unidades espaciales disímiles para explicar un mismo fenómeno, es decir, expresa diferencias en regiones geográficas (Anselin y Bera, 1998); esto se presenta de forma indistinta y particular en cada territorio, pues cada uno posee una relación distintiva (Acevedo-Bohórquez y VelásquezCeballos, 2008); en el comportamiento económico, esta particularidad genera patrones espaciales de desarrollo.

Para analizar este tipo de comportamiento el AEDE usa técnicas de visualización de distribuciones espaciales, usualmente acompañadas de métodos como gráficas capaces de identificar conexiones; combina herramientas de la estadística básica (descriptivas, correlaciones, tablas de frecuencias o de correlación cruzada) y cartográficas (diversas formas de presentación de mapas), así como enlaces entre la cartografía y los diferentes gráficos estadísticos, y tendencias o sesgos presentes en las bases de datos. Las técnicas más usadas para determinar la heterogeneidad espacial son mapa de cuantiles, box map, análisis de la varianza espacial, histograma de frecuencias y diagrama de dispersión (Acevedo-Bohórquez y Velásquez-Ceballos, 2008; Chasco-Yrigoyen, 2003b; Anselin y Bera, 1998).

El histograma muestra la frecuencia de las observaciones agrupadas y obtiene las medidas de tendencia central de los datos analizados: frecuencia, rango, media y desviación estándar (Chasco-Yrigoyen, 2009). Por su parte, los mapas consisten en la representación cartográfica de una variable geográfica que, mediante símbolos y colores, la ponen de manifiesto en cada una de las unidades geográficas consideradas (Chasco-Yrigoyen, 2009); se enfocan en resaltar los valores atípicos en los datos (Anselin, Syabri y Kho, 2006) espaciales, concentraciones de datos cuyo valor se encuentra a cierta distancia de la tendencia general (mediana); en los usados en el AEDE, los datos se dividen y agrupan en una serie de categorías (cuantiles) con igual número de observaciones (Chasco-Yrigoyen, 2009).

Por otro lado, se encuentra la llamada dependencia espacial, que ayuda a identificar si los fenómenos implican una relación funcional, es decir, asume valores similares condicionados por el mismo fenómeno en los territorios cercanos o vecinos; representa el más importante de los efectos espaciales y estudia la dependencia producida entre las obser- 
vaciones de una variable para sus diferentes localizaciones (ChascoYrigoyen, 2009; Aroca-González, 2000).

En el análisis de datos agregados geográficamente es frecuente encontrar que los valores de las variables estén autocorrelacionados espacialmente. La autocorrelación o dependencia espacial se define como la concentración o dispersión de los valores de una variable en un territorio, por tanto, refleja el grado en que actividades de una unidad geográfica resultan similares a otras actividades en sitios próximos, pues se encuentra condicionado por el valor que esa variable asume en una región vecina (Anselin, 1988; Goodchild, 1987). Para el análisis de dependencia espacial las técnicas usadas por el AEDE son el índice de Moran, el diagrama de dispersión de Moran y los mapas de LISA (Chasco-Yrigoyen, 2009).

\section{1. Índice de Moran}

Para verificar la existencia o ausencia de autocorrelación espacial, tanto Acevedo-Bohórquez y Velásquez-Ceballos (2008) como ArocaGonzález (2000) concuerdan en que el índice más usado es el de Moran (Moran, 1950): ofrece una medida resumen de la intensidad de la autocorrelación de los territorios considerados, el cual sólo puede ser utilizado en un análisis que incluya un conjunto de datos pequeños (Anselin, 1994); a su vez, permite atisbar los procesos de interrelación espacial de la variable analizada. Sin embargo, este índice muestra una perspectiva global del fenómeno de autocorrelación espacial, por lo que se le conoce como índice (I) global de Moran y tiene por objeto el contraste de la presencia de tendencias espaciales generales en la distribución de una variable sobre un espacio geográfico completo. Anselin (1995) propone el I estadístico local de Moran, cuyo fenómeno de dependencia local se define por una concentración, en un lugar del espacio analizado, de valores especialmente altos, bajos o atípicos de una variable, en comparación con su valor medio (Chasco-Yrigoyen, 2003a).

Dado que el I local es igual al I global por un factor de proporcionalidad (Chasco-Yrigoyen, 2003a), este estadístico también busca contrastar la hipótesis nula de ausencia de autocorrelación espacial, en este caso, para el supuesto inferencial de aleatoriedad (ChascoYrigoyen, 2003a); es decir, la variable analizada se encuentra distribuida de forma aleatoria entre las unidades de estudio. El I de Moran 
se mueve entre los valores -1 y +1 ; cuanto más cercano a 1 , mayor será el nivel de autocorrelación espacial. Si el valor es positivo, un fenómeno que tiene lugar en un territorio condiciona y favorece que se produzca en otro contiguo; por el contrario, la autocorrelación negativa se produce cuando el evento en una región limita su aparición en una colindante (Aroca-González, 2000; Anselin y Bera, 1998). Sin embargo, para el caso del I local de Moran se identifica la presencia de datos extremos que evidencian la importancia de esa observación en el estadístico global.

Para su obtención, Moran (1950) propone el siguiente índice:

$$
I=\frac{n}{S_{0}} \frac{\sum_{i=1}^{n} \sum_{j=1}^{n} W_{i j}\left(x_{i}-\bar{x}\right)\left(x_{j}-\bar{x}\right)}{\sum_{i=1}^{n}\left(x_{i}-\bar{x}\right)^{2}} \text { para } \mathrm{i}=\mathrm{j}
$$

Donde $\mathrm{W}_{\mathrm{ij}}$ es la matriz de pesos espaciales y simboliza la composición de la relación espacial entre distintos puntos; además, permite relacionar una variable en un punto en el espacio con sus propias observaciones en otras unidades espaciales (Acevedo-Bohórquez y Velásquez-Ceballos, 2008). La matriz es simétrica, transpuesta y, por lo tanto, cuadrada; sus elementos Wij representan la interdependencia existente entre las regiones i y j, serán no estocásticos y exógenos al modelo (Anselin, 1994); para la realización de esta investigación se usó la matriz de pesos espaciales tipo reina (Aroca-González, 2000).

Mientras, para obtener el I local de Moran (Anselin, 1995) es necesario agregar el factor de proporcionalidad $\gamma=\mathrm{S}_{0} \mathrm{~m}_{2}$, por lo que el índice se expresa de la siguiente forma:

$$
\sum I=\frac{1}{S_{0} m_{2}} * I=\gamma * I
$$

Donde $\mathbf{m}_{2}=\mathbf{\Sigma} \frac{Z_{i}^{2}}{N}$, un término constante en todas las localidades.

\subsection{Diagrama de Moran}

Una vez construida la matriz de pesos espaciales se procede al cálculo del índice, representado gráficamente mediante un diagrama de dispersión conocido como diagrama de Moran, obtenido a partir de la expresión matricial del I (Aroca-González, 2000); en él se representan la variable estandarizada en el eje X y el retardo espacial ${ }^{2}$ en el eje Y; la 
pendiente de la línea de regresión corresponde al I de Moran (Anselin, Syabri y Kho, 2006).

Este diagrama (Figura 1) muestra cuatro tipos de conglomerados espaciales, dos de autocorrelación positiva y los otros, negativa (ChascoYrigoyen, 2009), distribuidos en cuadrantes: el primero y el tercero (alto-alto, bajo-bajo) indican la presencia de autocorrelación positiva, es decir, el comportamiento de una variable en algunas regiones es favorecido por su comportamiento en zonas próximas; por otro lado, el segundo y cuarto cuadrantes (bajo-alto, alto-bajo) reflejan la existencia de autocorrelación espacial negativa, dado que el comportamiento de un fenómeno no está relacionado con efectos en los territorios vecinos (Aroca-González, 2000; Anselin, 1994).

Figura 1

DIAGRAMA DE DISPERSIÓN DE MORAN

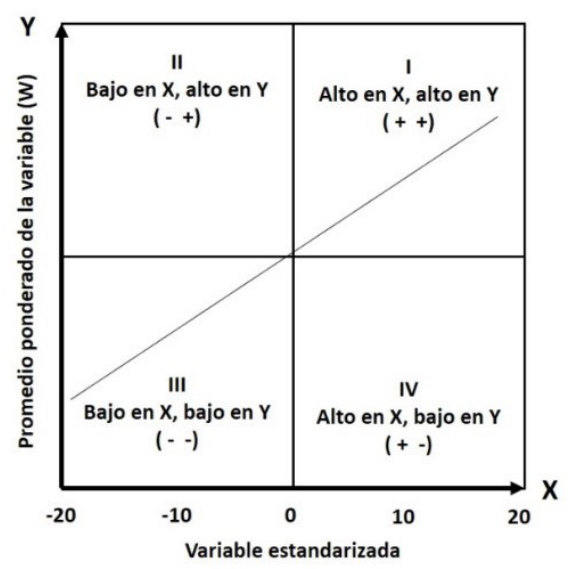

Fuente: Chasco-Yrigoyen (2003a).

Se tiene, entonces:

i) Alto-alto: una unidad territorial con un valor de análisis por encima del promedio, rodeada significativamente por áreas vecinas que también

2 Se entiende por retardo espacial el promedio ponderado de los valores que adopta una variable en el subconjunto de observaciones vecinas (Chasco-Yrigoyen, 2009). 
se encuentran sobre la media con respecto a la variable de interés; estas unidades territoriales corresponden a los denominados conglomerados calientes (hot spots);

ii) bajo-alto: una unidad territorial con un valor de análisis bajo, rodeada significativamente por áreas vecinas con valores por sobre la media de la variable de interés;

iii) bajo-bajo: una unidad territorial con un valor de análisis inferior al promedio, rodeada por áreas vecinas que también se encuentran bajo la media en relación con la variable de interés; estas unidades territoriales corresponden a los denominados conglomerados fríos (cold spots);

iv) alto-bajo: una unidad territorial con un valor de análisis alto, rodeada significativamente por áreas vecinas con valores bajo la media de la variable de interés.

\subsection{Mapa de LISA}

Por último, para la determinación de la existencia del clúster, o asociación espacial, se obtiene el mapa de LISA, donde se representan las localizaciones con valores significativos en determinados indicadores estadísticos de asociación espacial local y mide el grado de concentración de valores altos y bajos de una variable en el entorno geográfico de cada observación de la muestra; se identifican puntos calientes (hot spots) o atípicos espaciales (Chasco-Yrigoyen, 2009). Los valores altos representarían un clúster espacial donde existe la ocurrencia de un evento con distribución homogénea (Moreno-Serrano y Vayá-Valcarse, 2000).

\section{EL AEDE Y DISCUSIÓN DE RESULTADOS}

\subsection{Características socioeconómicas del Estado de México}

Ubicada en la región centro-sur del país, el Estado de México limita al norte con Querétaro, al noreste con Hidalgo, al este con Tlaxcala, al sureste con Puebla, al sur con Morelos y Ciudad de México, al suroeste con Guerrero y al oeste con Michoacán (INEGI, 2015c), por lo que cuenta con una incomparable accesibilidad al mayor mercado a nivel nacional, así como a los diferentes puntos de enlaces comerciales del país (ver Mapa 1). Se localiza geográficamente entre los paralelos $18^{\circ}$ 
$21^{\prime}$ y $20^{\circ} 17^{\prime}$ de latitud norte y $98^{\circ} 36^{\prime}$ y $100^{\circ} 36^{\prime}$ de longitud oeste, a una altura de 2683 metros sobre el nivel del mar en su planicie más elevada, el Valle de Toluca (Inafed, 2010).

Mapa 1

UBICACIÓN GEOGRÁFICA DEL ESTADO DE MÉXICO

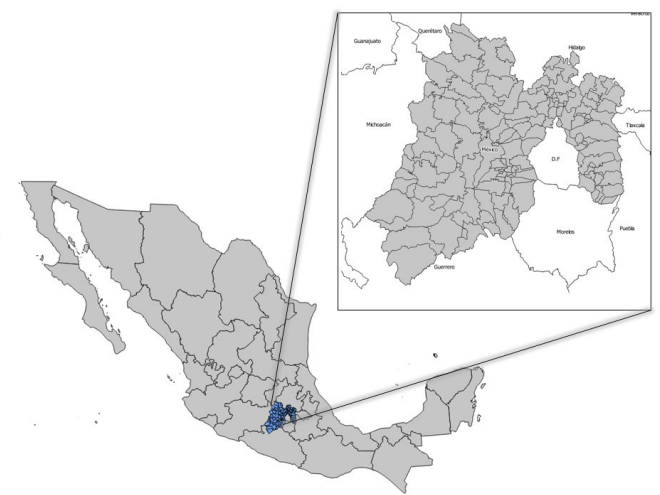

Fuente: Marco Geo estadístico Ver. 6.5 (INEGI, 2015c).

Esta entidad ocupa el lugar 25 en extensión territorial (Inafed, 2010) con $22357 \mathrm{~km}^{2}$, lo cual representa $1.14 \%$ del total del país (INEGI, 2015c). Una de sus principales fortalezas es la red carretera -tercera en la República (Imco, 2016) con más de 14 mil kilómetros- y cuenta con más de mil 400 kilómetros de vías férreas, dedicadas a interconectar a cerca de un centenar de parques industriales, es decir, 15\% del total del país. El Estado de México es un punto logístico estratégico, el enlace entre los principales puertos marítimos del oriente y occidente del país; asimismo, cuenta con un Aeropuerto Internacional, localizado en Toluca.

Es el estado más poblado del país con 16,187,608 habitantes, lo que representa $13.5 \%$ de la población nacional (INEGI, 2015a); ha presentado un crecimiento promedio anual de $2.23 \%$ en los últimos 12 años (INEGI, 2015a). En su interior es evidente la heterogeneidad en la forma como se distribuye la población, pues se concentra en las zonas urbanas aledañas a la capital de la República; en ese sentido, 20\% de los municipios de esta entidad (25) son los más poblados: concentran $74 \%$ de la población. De estos municipios sobresalen once: Nezahualcóyotl, Naucalpan, Tlalnepantla, Chimalhuacán, Cuautitlán Izcalli, Atizapán de Zaragoza, Tultitlán, Ixtapaluca, Tecámac y Nicolás Romero que, junto 
con Toluca, concentran 54\% del total de los mexiquenses. En contraste, cien municipios aglutinan menos de 156,000 habitantes, lo que representan 26\% de la población estatal (INEGI, 2015a). En este sentido, los dos municipios cuyos valores resultan los más contrastantes son Otzoloapan (con el menor número de habitantes de la entidad: 3,872 ) y Ecatepec (el más poblado a nivel estatal y el segundo a nivel nacional con 1,677,678 habitantes).

La actividad económica del Estado de México está sustentada tanto en la industria como en el sector terciario: en 2015, la primera representó 30.85\% del PIB estatal y los servicios, 65.18\% (IGECEM, 2016); ambos presentan un patrón de concentración similar al de la población: Toluca y la zona conurbada de la Ciudad de México. Once municipios tienen una participación muy importante en el PIB estatal, al concentrar $76 \%$ de toda la economía de la entidad: Toluca, Metepec, Huixquilucan, Atizapán de Zaragoza, Tlalnepantla, Ecatepec, Nezahualcóyotl, La Paz, Tultitlán, Cuautitlán Izcalli y Naucalpan; tan solo este último representa $18.51 \%$ del PIB estatal (IGECEM, 2016; INEGI, 2015b).

\subsection{Variables y fuentes de información}

En este análisis se usan datos de corte transversal de los 125 municipios del Estado de México correspondientes a 2010, para cada una de las variables del IDH; se estudian éstas a través del histograma, el mapa de cuartiles y el box map.

Para determinar el nivel de salud se emplea la tasa de mortalidad infantil: el número de defunciones de menores de un año de edad por cada mil nacimientos ocurridos (Conapo, 2013); para establecer el nivel educativo se utilizan los años promedio de escolaridad: media de los acumulados de educación de todas las personas mayores a 24 años a nivel municipal (INEGI, 2010), así como los esperados de escolarización: población que asiste a la escuela entre 6 y 24 años entre la población de 6 a 24 años (INEGI, 2010); por último, para el nivel de ingreso se considera el ingreso per cápita anual (dólares estadounidenses PPC), ajustado a cuentas nacionales (PNUD, 2014). Para el caso del IDH municipal 2010, se toma en cuenta el presentado por PNUD (2014), obtenido una vez que se calculan los índices de cada una de las capacidades: se agrupan y se utiliza una media geométrica, que representa que no existe sustituibilidad perfecta entre los diferentes aspectos del desarrollo humano. 


\subsection{Nivel de salud en los municipios del Estado de México} El nivel de salud, contemplado por el PNUD para la construcción del IDH, está representado por la mortalidad infantil como condición de esperanza de vida al nacer, a partir del número de defunciones de menores de un año por cada mil nacimientos ocurridos en el año 2010 (Conapo, 2013).

Al realizar un análisis de heterogeneidad, se observa que el Estado de México presenta una media de 17.56 defunciones por cada mil habitantes; la gráfica de distribución de frecuencia muestra un sesgo hacia la izquierda como indicativo de que existen más municipios con menos defunciones (96 de 125). Pese a los esfuerzos por combatir este fenómeno, hay ocho municipios con más de 23.52 defunciones por cada mil habitantes: Ecatzingo, Sultepec, Morelos, Ocuilan, San José del Rincón, San Felipe del Progreso, Joquicingo y Villa de Allende, dato mostrado en tono obscuro en la Gráfica 1 (Conapo, 2013).

Gráfica 1

ESTADO DE MÉXICO, TASA DE MORTALIDAD INFANTIL (2010)

HISTOGRAMA DE FRECUENCIA

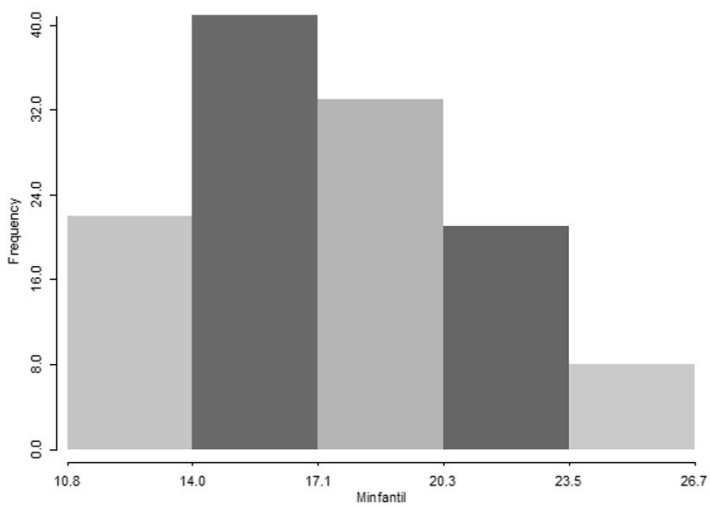

Fuente: Elaboración propia con datos de Conapo (2013).

El diagrama de caja expresa que no existen datos atípicos (outliers), mientras en el mapa de percentiles (Mapa 2) se muestra con color más obscuro, en el norte, a Morelos, el municipio con la mayor tasa de mortalidad infantil (26.71), y de color más claro, a San Antonio la Isla como el de menor tasa con 10.76 (Conapo, 2013). 
El estudio supone la dispersión de este fenómeno en el territorio estatal: de los doce municipios con mayor índice, cuatro se localizan al norte; tres, al oeste; dos más, al sur y uno está en el extremo sureste de la entidad (Mapa 2). Esta condición de dispersión se confirmará al analizar la autocorrelación espacial.

\section{Mapa 2}

TASA DE MORTALIDAD INFANTIL, ESTADO DE MÉXICO (2010)

MAPA DE PERCENTILES Y DIAGRAMA DE CAJA

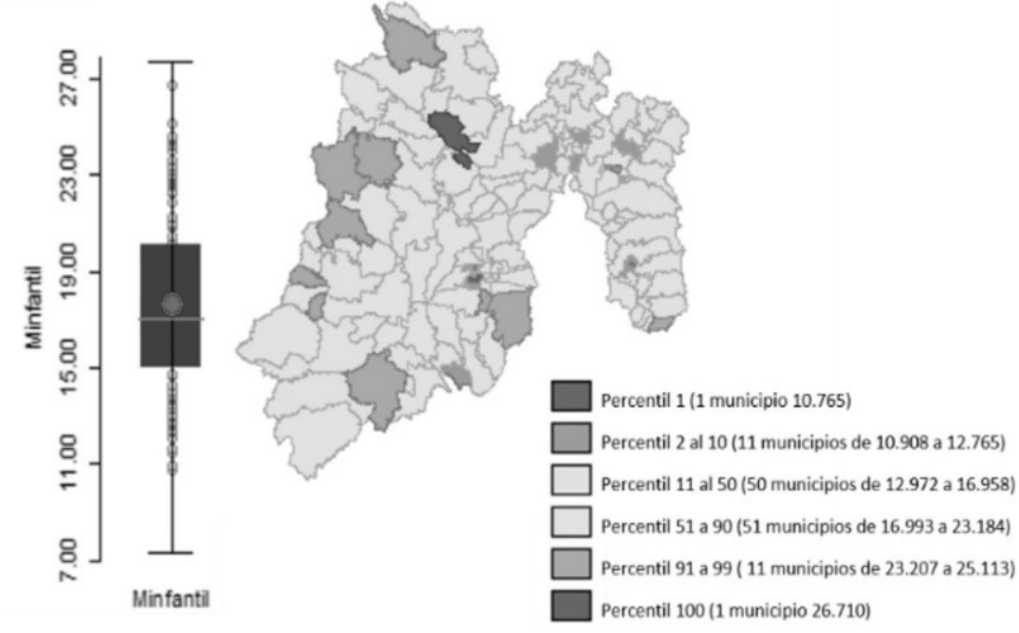

Fuente: Elaboración propia con datos de Conapo (2013).

\subsection{Nivel de educación en los territorios del Estado de}

\section{México}

En primer lugar, se abordó el grado de estudios de esta entidad: 7.104 años, en promedio, dos años por debajo de la media nacional, que es de 9.1 (INEGI, 2015c). El histograma de frecuencia de este indicador presenta un sesgo a la izquierda, lo que significa que existen más municipios con menos años de escolaridad que la media estatal; tal rango implica un fenómeno preocupante: 7.8 años de diferencia, como se representa en la Gráfica 2 (INEGI, 2010).

El municipio con superior nivel educativo es Metepec (11.815 años), de color más obscuro en el Mapa 3; pese a no ser considerado un outlier, destaca en la parte superior del diagrama de caja, con 1.3 años de escolaridad más que el segundo lugar estatal (Coacalco de Berrio- 
zábal). En el extremo contrario se localiza Luvianos, al suroeste de la entidad, con 3.9 años (de color más claro), lo que implica que los habitantes de este territorio solo tienen la mitad del nivel básico de educación, muy por debajo de la mitad del promedio de instrucción nacional: educación básica y media terminada (INEGI, 2010).

Gráfica 2

AÑoS DE ESCOLARIDAD PROMEDIO ESTAdO DE MÉXICO (2010)

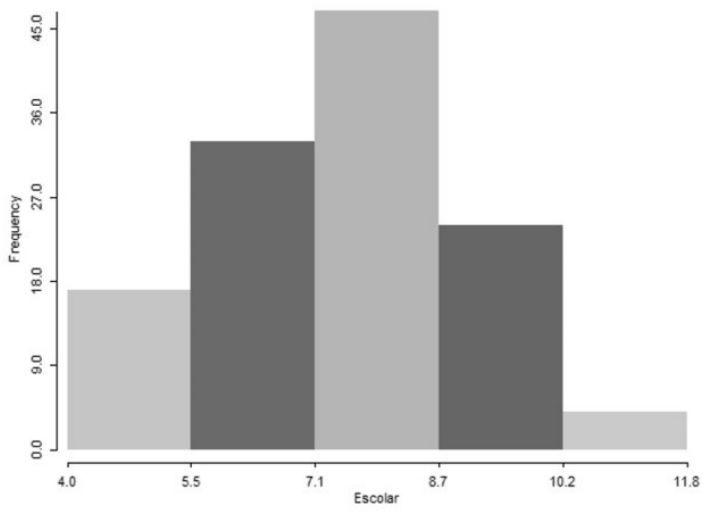

Fuente: Elaboración propia con datos de INEGI (2010).

De acuerdo con el análisis de heterogeneidad espacial, se presume la presencia de autocorrelación pues existen dos territorios que concentran altos niveles de educación, representados en el Mapa 3 en obscuro, y una región al oeste de la entidad donde persisten bajos niveles de este indicador (en claro); esto se confirmará más adelante mediante la obtención del índice de Moran.

Asimismo, se muestran los años esperados de escolaridad: incluye la población que asiste a la escuela entre 6 y 24 años; el histograma de frecuencias refleja una distribución normal, concentrado en torno a la media, 12.03 años en promedio, lo que a futuro representaría una mejora sustantiva de las capacidades de la población (INEGI, 2010). No existe mucha dispersión de los datos pues la desviación estándar es poco menor a un año (0.9689), como se expresa en la Gráfica 3. 
Gráfica 3

ESPERANZA EN AÑOS DE ESCOLARIDAD PROMEDIO ESTADO DE MÉxICO (2010) HISTOGRAMA DE FRECUENCIA

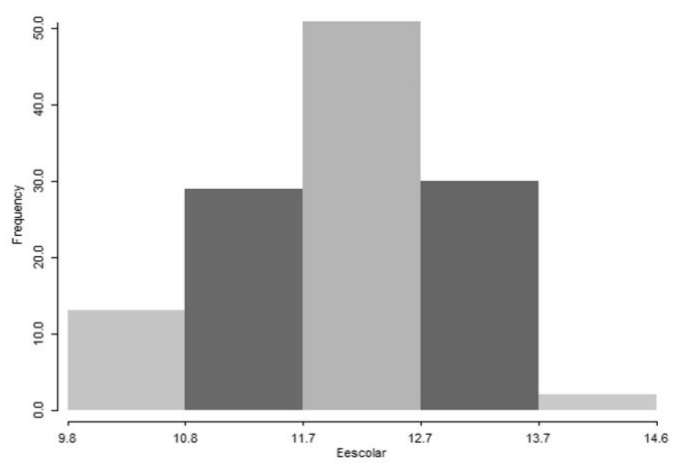

Fuente: Elaboración propia con datos de INEGI (2010).

Este indicador sí presenta un outlier, punto atípico que sale en la parte superior del diagrama de caja: Metepec, el municipio (en obscuro) con más esperanza de años de escolaridad con 14.635, es decir, su población contaría con un nivel de enseñanza profesional (INEGI, 2010). En sentido contrario, el municipio que menos esperanza educativa muestra es Coatepec Harinas, localizado al sur del estado (en claro), con el mismo nivel promedio de educación que tiene el país actualmente: 9.7 años (ver Mapa 4).

Mapa 3

AÑOS DE ESCOLARIDAD PROMEDIO ESTADO DE MÉXICO (2010)

MAPA DE PERCENTILES Y DIAGRAMA DE CAJA

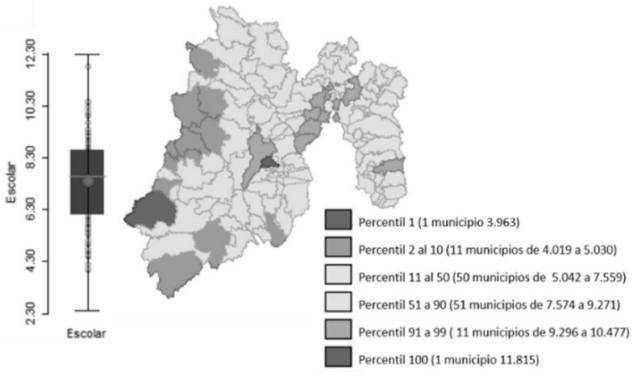

Fuente: Elaboración propia con datos de INEGI (2010). 


\section{Mapa 4}

ESPERANZA EN AÑOS DE ESCOLARIDAD PROMEDIO ESTAdo DE MÉxICO (2010)

MAPA DE PERCENTILES Y DIAGRAMA DE CAJA

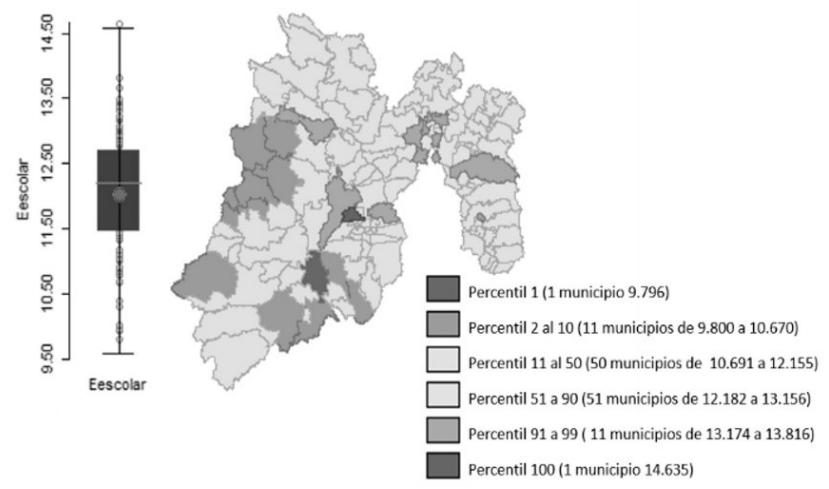

Fuente: Elaboración propia con datos de INEGI (2010).

La heterogeneidad de este fenómeno supone una concentración espacial pero solo de los datos más bajos de esperanza de educación, presentes en el oeste de la entidad, mientras que los municipios con mayor esperanza de nivel de educación se distribuyen heterogéneamente; esto se confirmará o rechazará más adelante.

\subsection{Nivel de ingresos en los municipios del Estado de México}

El análisis de heterogeneidad de este indicador confirma la existencia de un problema en la distribución del ingreso al interior del territorio, cuyo promedio es de 10,274.7 dólares anuales (PNUD, 2014); asimismo, presenta una desviación estándar de menos de la mitad de la media: 4,019.96 dólares, lo cual implica una diferencia notable entre los municipios con mayor y menor ingreso. Lo anterior se confirma en el histograma de frecuencia, donde se visualiza la presencia de 75 municipios con menos ingresos de la media (60\% del total estatal), representada por una asimetría considerable con un sesgo a la izquierda (Gráfica 4). Por otra parte, se observa la presencia de seis municipios con un ingreso superior a los 20,028.4 dólares: Atizapán de Zaragoza, Coacalco, Huixquilucan, Naucalpan y Cuautitlán, localizados en la zona conurbada del Valle de México, así como Metepec, marcado en obscuro (Mapa 5). 


\section{Gráfica 4}

NiVEL DE INGRESOS PER CÁPITA ESTAdo de MÉXICO (2010)

HISTOGRAMA DE FRECUENCIA

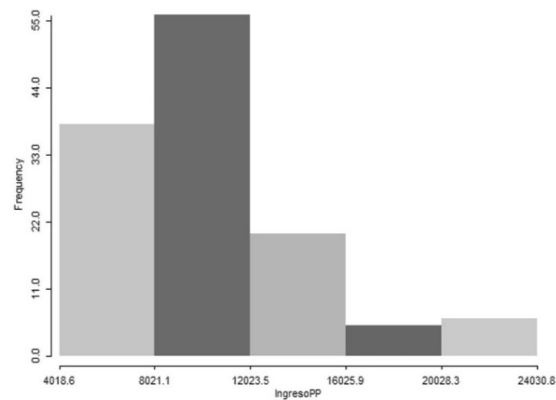

Fuente: Elaboración propia con datos de PNUD (2014).

En este indicador se observa una cantidad importante de outliers, representados como puntos en la parte superior del diagrama de caja (Mapa 5): Atizapán de Zaragoza, Coacalco, Ixtapaluca, Tecámac, Huixquilucan, Naucalpan, Cuautitlán, Tlalnepantla, Metepec, Cuautitlán Izcalli y Toluca. Metepec es el de mayor ingreso, con 24,030.76 dólares anuales; por el contrario, San José del Rincón, localizado al oeste de la entidad y marcado en tono claro, cuenta con el ingreso más precario, que es de 4,018.63 dólares (PNUD, 2014).

\section{Mapa 5}

NiVEL DE INGRESOS PER CÁPITA ESTADO DE MÉXICO (2010)

MAPA DE PERCENTILES Y DIAGRAMA DE CAJA

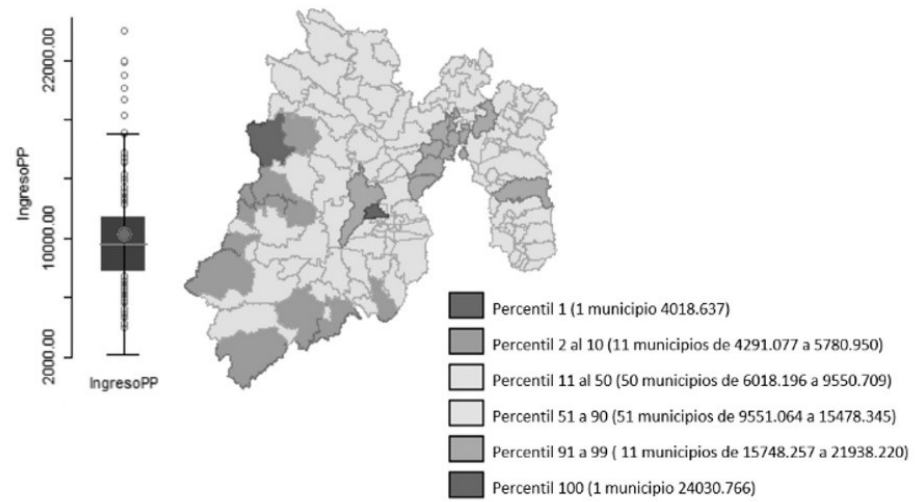

Fuente: Elaboración propia con datos de PNUD (2014). 
La heterogeneidad en las ganancias muestra claramente la presencia de una zona de altos ingresos en las regiones conurbadas de la Ciudad de México y de la ciudad de Toluca, en contraste con otra zona de los más bajos localizada en el oeste y sur de la entidad.

\section{6. Índice de Desarrollo Humano en los municipios del Estado de México}

A diferencia del ingreso, el IDH se distribuye de forma más homogénea con una media de 0.6901 y una desviación estándar pequeña de 0.05933 , lo que significa que su media se concentra en torno a 83 municipios, es decir, implica un nivel moderado de desarrollo (entre 0.633077 y 0.74943 ); por ello, en el box map se encuentra en el centro (Mapa 6), con un ligero sesgo a la derecha en su histograma de frecuencias, como se aprecia en la Gráfica 5 (PNUD, 2014).

Sin embargo, esto no significa que las condiciones de desarrollo resulten equitativas para los mexiquenses, sino que existe igual cantidad de municipios con alto grado de progreso, medido por el IDH, que con uno menor; esto se visualiza en el Mapa 6 con color claro, repartido en las zonas sur, oeste y norte de la entidad (PNUD, 2014).

Gráfica 5

Í́ndice de Desarrollo Humano Estado de México (2010)

HISTOGRAMA DE FRECUENCIA

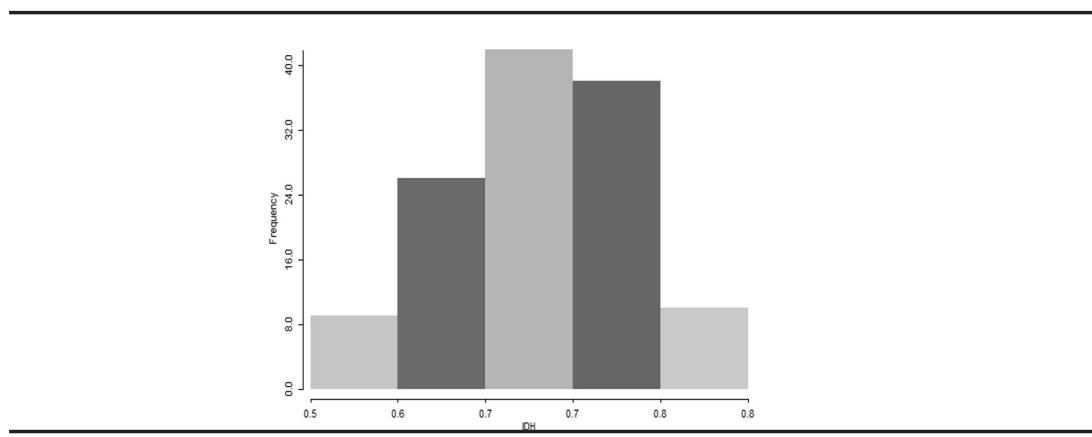

Fuente: Elaboración propia con datos de PNUD (2014).

La heterogeneidad en el Estado de México se torna evidente entre los municipios aledaños a la Ciudad de México y la capital mexiquense, y los más alejados de esta zona. Los primeros, localizados en los polos de atracción económica y, por consiguiente, poblacional, se ven beneficiados por las externalidades propias de su ubicación. Se observa ese mismo fenómeno al analizar el IDH de los municipios que conforman 
la entidad: doce exhiben mayores índices de desarrollo; Metepec es el más alto con un IDH de 0.825 (no llega a representar un dato atípico), seguido por Coacalco, Cuautitlán Izcalli, Cuautitlán, Atizapán de Zaragoza, Tlalnepantla, Tecámac, Naucalpan, Huixquilucan, Nezahualcóyotl y Tultitlán, señalados con color obscuro (PNUD, 2014), situados en la zona conurbada de la Ciudad de México; separado de esta región se encuentra Toluca, asentado junto a Metepec (Mapa 7).

En contraste, alejados de los polos de desarrollo económico, se presentan los municipios con bajo IDH, en color claro, dispersados en dos zonas: al sur de la entidad, Tlatlaya, Zumpahuacán, Zacualpan, Sultepec y Luvianos; al oeste, Amanalco, Ixtapan del Oro, Donato Guerra, Villa Victoria y Villa de Allende, los cuales se distinguen en el Mapa 7 con un azul zafiro. Consideración especial amerita el caso de San José del Rincón (en azul obscuro), que presenta el IDH más bajo de la entidad con 0.545 (PNUD, 2014).

\section{Mapa 6}

Índice de Desarrollo Humano Estado de MÉxico (2010)

MAPA DE PERCENTILES Y DIAGRAMA DE CAJA

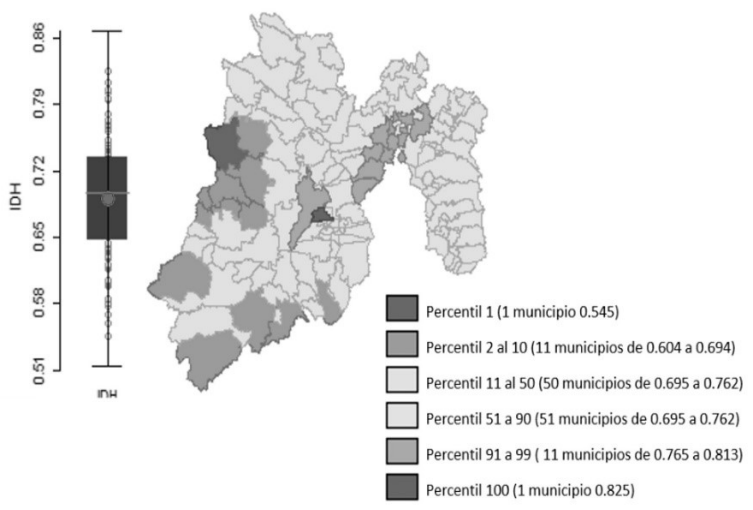

Fuente: Elaboración propia con datos de PNUD (2014).

Lo anterior refuerza la idea de que la concentración de la economía y de la población determina del desarrollo de algunos municipios del Estado de México, lo que sin duda abre la posibilidad de profundizar el análisis.

\subsection{Dependencia espacial}

El resultado del test para el I local de Moran, aplicado a los indicadores antes descritos, rechaza la hipótesis de la existencia de aleatoriedad 
espacial; muestra una alta correlación espacial positiva en educación: 0.6929 para años de escolaridad (Gráfica 7) y 0.6051 para esperanza de años de escolaridad (Gráfica 8), además de 0.5618 para el nivel de ingresos (Gráfica 9). Ello se representa visualmente dentro de los diagramas de dispersión de Moran, al concentrarse los puntos en torno a la línea recta; como la mayoría se aglutina en los cuadrantes I (altoalto), se tienen hot spots, es decir, unidades territoriales por encima del promedio que cuentan con vecinos también superiores a la media; por otra parte, se localizan en el cuadrante III (bajo-bajo) cold spots, unidades territoriales con valores inferiores al promedio, rodeada por áreas vecinas de la misma condición.

En el caso de la mortalidad infantil, pese a que existe correlación espacial positiva, no resulta contundente: tiene un I local de Moran de 0.1767 (Gráfica 6), de manera que los puntos se encuentran dispersos en los cuatro cuadrantes, no concentrados entorno a la línea recta, evidencia de que este fenómeno se presenta aleatoriamente en el territorio.

Gráfica 6

MORTALIDAD INFANTIL, ESTADO DE MÉXICO (2010)

DIAGRAMA DE MORAN

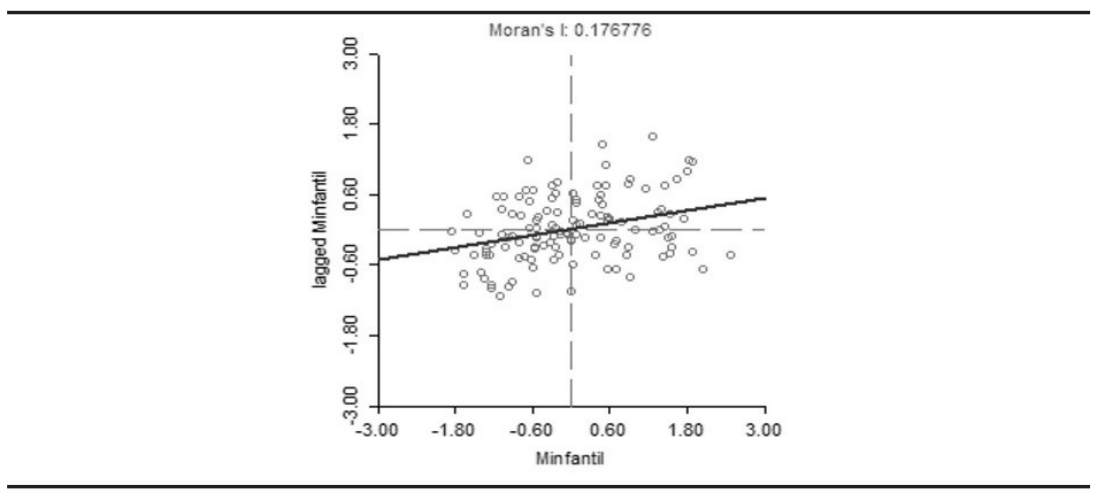

Fuente: Elaboración propia con datos de Conapo (2013). 


\section{Gráfica 7}

AÑos DE ESCOLARIDAD, ESTADO DE MÉxICo (2010)

DIAGRAMA DE MORAN

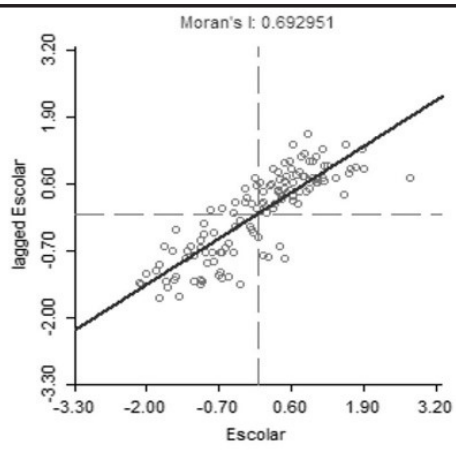

Fuente: Elaboración propia con datos de INEGI (2010).

\section{Gráfica 8}

ESPERANZA DE AÑOS DE ESCOLARIDAD, ESTADO DE MÉXICO (2010)

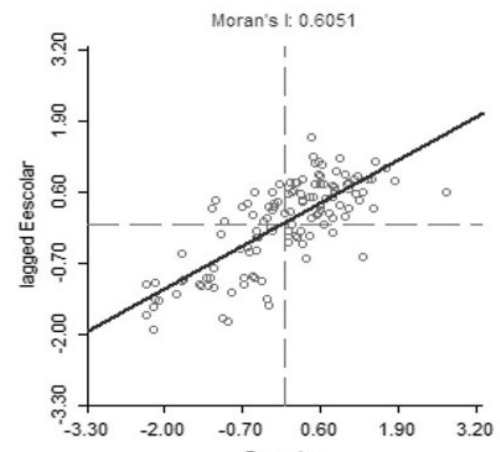

Eescolar

Fuente: Elaboración propia con datos de INEGI (2010). 
Gráfica 9

INGRESO PER CÁPITA, ESTAdO DE MÉXICO (2010)

DIAGRAMA DE MORAN

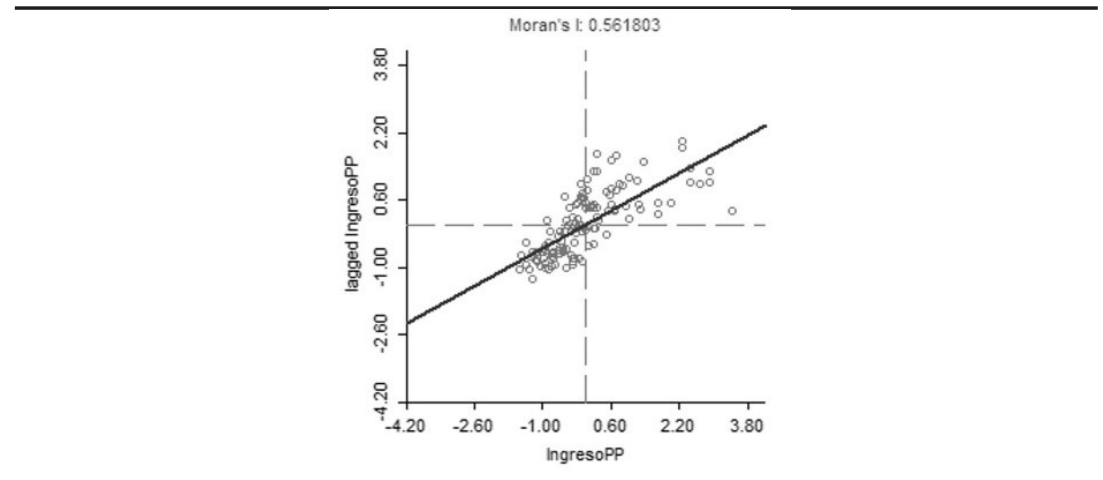

Fuente: Elaboración propia con datos de PNUD (2014).

Finalmente, se encontró que el I local de Moran para el IDH en los municipios mexiquenses fue de 0.6493 (Gráfica 10), lo que demuestra que para la entidad el bajo o alto desarrollo humano no se distribuye de manera aleatoria. En este caso, existen hot spots y cold spots dentro de los cuadrantes I y III, los cuales representarían clústers perfectamente definidos de territorios con alto y bajo IDH, cuya autocorrelación espacial implica condiciones de vecindad con municipios de IDH semejante.

\section{Gráfica 10}

ÍNDICE de DESARRollo Humano, ESTAdo de MÉxICO (2010)

DIAGRAMA DE MORAN

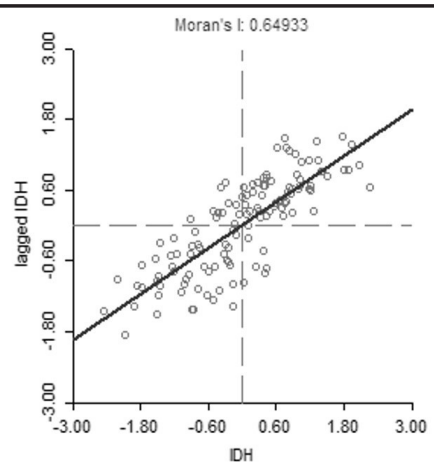

Fuente: Elaboración propia con datos de PNUD (2014).

La disposición espacial de los clústers observados en los diagramas de dispersión de Moran es presentada mediante los mapas de LISA; 
aquellos en color azul son los cold spots del cuadrante bajo-bajo, y en color rojo están los hot spots del cuadrante alto-alto. Resultan más evidentes los de puntos con el índice de Moran más alto, a saber, educación, ingresos e IDH.

A continuación se presentan los municipios que conforman los clústers obtenidos a partir del análisis de autocorrelación espacial y sus respectivos mapas de LISA.

\section{Mapa 7 \\ AÑos DE ESCOLARIDAD, ESTADO DE MÉXICO (2010) \\ MAPA DE LISA}

Municipios integrantes del clúster bajo-bajo

El Oro, Sultepec, Almoloya de Alquisiras, Coatepec Harinas, San Simón de Guerrero, Amatepec, Temascaltepec, Villa Victoria, Otzoloapan, Donato Guerra, Amanalco, Luvianos, Valle de Bravo, Texcaltitlán, Timilpan, San José del Rincón, Zacazonapan, San Felipe del Progreso, Ixtapan de la Sal, Tejupilco, Santo Tomas, Villa de Allende, Zacualpan.

\section{Municipios integrantes del clúster alto-alto}

Atizapán de Zaragoza, Ocoyoacac, Mexicaltzingo, Zumpango, Ixtapaluca, Tecámac, Jaltenco, Nextlalpan, Atenco, Naucalpan, Cuautitlán, Tlalnepantla, San Mateo Atenco, Ecatepec, Tonanitla, Melchor Ocampo, Texcoco, Metepec, Tultitlán, Chiautla, Tultepec, Tlalmanalco, Teoloyucan, Lerma, Calimaya, CuautitIán Izcalli, Tepotzotlán, Tianguistenco, Tepetlaoxtoc, Chapultepec.

Fuente: Elaboración propia con datos de INEGI (2010).

Puede notarse, en el Mapa 7, la presencia de los cold spots en las regiones oeste y sur del estado, donde se localizan los territorios con menos años de escolaridad promedio; este clúster supone la existencia de territorios y zonas aledañas que comparten la misma condición. Por otro lado, en el área conurbada de la Ciudad de México se localizan los hot spots, donde los municipios presentan alto nivel educativo, como sus vecinos. 
Mapa 8

ESPERANZA DE AÑOS DE ESCOLARIDAD, ESTADO DE MÉxICO (2010)

MAPA DE LISA

Municipios integrantes del clúster bajo-bajo

El Oro, Sultepec, Zumpanhuacán, Almoloya de Alqui- Atizapán de Zaragoza, Mexicaltzingo, Ixtapaluca, siras, Coatepec Harinas, Villa Guerrero, Malinalco, Tecámac, Huixquilucan, Jaltenco, Nextlalpan, AtenIxtapan del Oro, Temascaltepec, Villa Victoria, Otzoloapan, Donato Guerra, Tenancingo, Tonatico, Amanalco, Luvianos, Valle de Bravo, Texcaltitlán, San José del Rincón, Zacazonapan, Ixtapan de la Sal, Tejupilco, Santo Tomas, Villa de Allende, Zacualpan
Municipios integrantes del clúster alto-alto

Fuente: Elaboración propia con datos de INEGI (2010).

En el Mapa 8 se observa, como en el anterior, la existencia de los clústers en las mismas regiones, debido a que implican variables sobre el nivel de educación de cada municipio; como no se visualizan puntos relacionados con el cuadrante bajo-alto, se confirma que la esperanza en años de educación no se presenta de forma aislada en la entidad. 
Mapa 9

MoRTALIDAD INFANTIL, ESTADO DE MÉXICO, (2010)

MAPA DE LISA

Municipios integrantes del clúster bajo-bajo

Municipios integrantes del clúster alto-alto

1. San José del Rincón, Villa de Allende, Villa Victoria, Mexicaltzingo, Tecámac, Jaltenco, Nextlalpan, Tlal- Amanalco, Almoloya de Juárez, Zinacantepec. nepantla, Ecatepec, Tonanitla, Tultitlán, Tultepec, Cuautitlán.

2. Xalatlaco, Ocuilan, Malinalco, Tenancingo, Joquicingo.

Fuente: Elaboración propia con datos de Conapo (2013).

Debido a que el I local de Moran relativo a la mortalidad infantil es cercano a 0 (Gráfica 6), no se exhibe claramente la presencia de clústers, sin embargo, destacan tres: el bajo-bajo, al norte de la Ciudad de México, donde se presentan las tasas mínimas; y dos alto-alto, uno al sureste y otro al poniente, con tasas elevadas (Mapa 9). También existen puntos aislados, como Metepec, que no se consideran parte del clúster pues, aunque presentan bajas tasas de mortalidad infantil, no implican contigüidad con municipios de la misma condición (Conapo, 2013). 
Mapa 10

NiVel de INGRESOS, ESTAdo DE MÉxiCO, (2010)

MAPA DE LISA

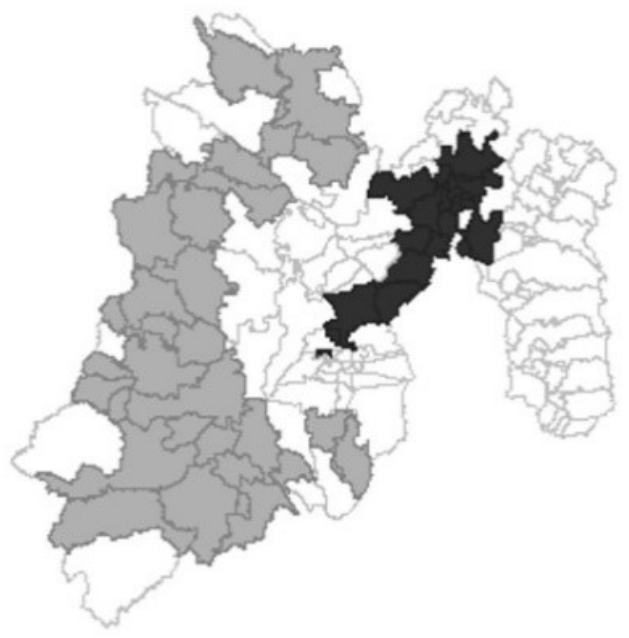

Municipios integrantes del clúster bajo-bajo

Municipios integrantes del clúster alto-alto

El Oro, Sultepec, Chapa de Mota, Jocotitlán, Almoloya

de Alquisiras, Coatepec Harinas, Jilotepec, Malinalco, Atizapán de Zaragoza, Mexicaltzingo, Coacalco, ZumAmatepec, Temascaltepec, Atlacomulco, Morelos, Vi- pango, Huixquilucan, Jaltenco, Nextlalpan, NaucalIla Victoria, Otzoloapan, Donato Guerra, Tenancingo, pan, Cuautitlán, Tlalnepantla, San Mateo Atenco, Amanalco, Valle de Bravo, Texcaltitlán, Timilpan, San Ecatepec, Tonanitla, Melchor Ocampo, Tultitlán, José del Rincón, Zacazonapan, San Felipe del Progre- Tultepec, Lerma, Chicoloapan, Cuautitlán Izcalli, Teso, Ixtapan de la Sal, Tejupilco, Villa de Allende, Za- potzotlán.

cualpan.

Fuente: Elaboración propia con datos de PNUD (2014).

El clúster bajo-bajo referente al nivel de ingresos presenta una particularidad: se ubica en la parte oeste de la entidad, de norte a sur, de manera que abarca un gran número de municipios; ello indica que, en esta región (con los municipios más alejados de la capital del país), la autocorrelación espacial define que los territorios con bajos niveles de ingresos están vinculados con vecinos en la misma condición. Por el contrario, los altos niveles de ingresos se aglutinan en la región metropolitana del Valle de México, donde se concentra la mayor dinámica económica de la entidad (Mapa 10). 


\section{Mapa 11}

Índice de DesarRollo humano, Estado de MÉxico (2010) MAPA DE LISA

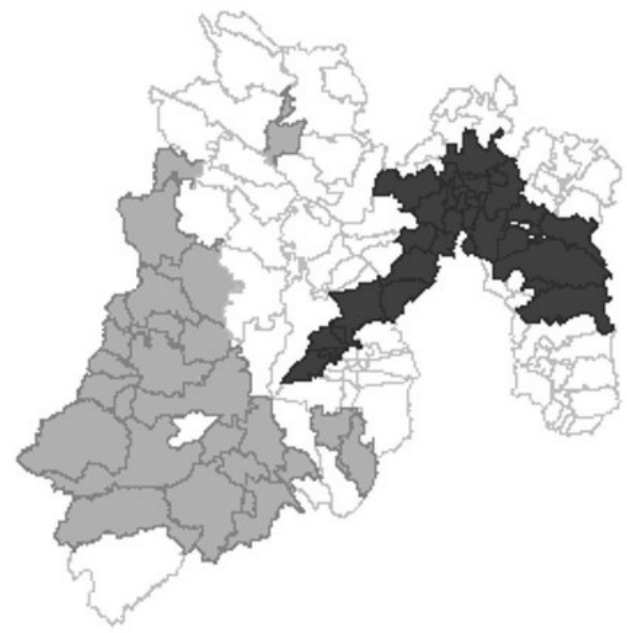

Atizapán de Zaragoza, Mexicaltzingo, Coacalco, Zum-

El Oro, Sultepec, Almoloya de Alqusiras, Coatepec Harinas, Malinalco, Ixtapan del Oro, Amatepec, Temascaltepec, Villa Victoria, Otzoloapan, Donato Guerra, Tenancingo, Amanalco, Luvianos, Valle de Bravo, Texcaltitlán, Timilpan, San José del Rincón, Ixtapan de la Sal, Tejupilco, Villa de Allende, Zacualpan. pango, Ixtapaluca, Tecámac, Jaltenco, Nextlalpan, Atenco, Naucalpan, Cuautitlán, Tlalnepantla, San Mateo Atenco, Ecatepec, Tonanitla, Melchor Ocampo, Texcoco, Metepec, Tultitlán, Chiautla, Tultepec, Acolman, Teoloyucan, Lerma, Calimaya, Chicoloapan, Cuautitlán Izcalli, Tepotzotlán, Tepetlaoxtoc, Chapultepec.

Fuente: Elaboración propia con datos de PNUD (2014).

Finalmente, el mapa de LISA sobre el IDH es mucho más parecido a los obtenidos para el nivel de educación, pues sugiere la presencia de un clúster bajo-bajo en las regiones oeste y sur de la entidad, con vecinos de iguales rasgos, así como la existencia de un gran corredor rojo de territorios con un IDH alto, cuya vecindad ayuda a que los más próximos tengan un IDH también alto (Mapa 11). 


\section{CONCLUSIONES}

Se ha presentado evidencia de la presencia de heterogeneidad espacial tanto en el IDH como en los componentes educación (años de escolaridad y años esperados de escolaridad) e ingresos en el Estado de México, lo que ha dejado vulnerables zonas específicas ubicadas en los clústers bajo-bajo, en contraste con los municipios que presentan altos niveles, localizados particularmente en la zona conurbada de la Ciudad de México.

Las externalidades proporcionadas, tanto por la ubicación geográfica como por la participación en la economía nacional, no han logrado permear en forma positiva en todos los territorios ubicados en esta entidad federativa. A lo largo de este trabajo se ha demostrado la existencia de una zona muy vulnerable en las regiones sur y oeste del Estado de México, lejanas a los polos de atracción económica, por lo que no logran aprovechar su inercia (IGECEM, 2016); se trata de los municipios con el menor nivel de desarrollo humano: San José del Rincón, Amanalco, Donato Guerra e Ixtapan del Oro, al oeste; Almoloya de Alquisiras, Amatepec, Luvianos, Sultepec y Zacualpan, al sur.

Dada la presencia de clústers en educación, se deben impulsar políticas tendientes a elevar el nivel educativo en los municipios señalados como cold spots (al oeste y sur), pues resulta inconcebible la existencia de sitios con un nivel educativo tan bajo como Luvianos, con 3.9 años. En lo particular se pone de manifiesto la existencia de 25 municipios vecinos con un nivel bajo de educación, condición que no parece mejorar en el futuro, pues el clúster bajo-bajo sobre esperanza de educación es de 24 municipios; esto podría explicarse por la existencia de más municipios con una escolaridad menor a la media estatal, en particular, seis presentan el nivel más bajo: Luvianos, San José del Rincón, Sultepec, Otzoloapan, Ixtapan del Oro y Villa de Allende, ubicados en la región oeste de la entidad. Por el contrario, aquellos con mayor nivel de educación superan los diez años de escolaridad promedio: Huixquilucan, Atizapán de Zaragoza, Cuautitlán, Cuautitlán Izcalli, Coacalco de Berriozábal, en la zona conurbada con la Ciudad de México, mientras que Metepec representa un dato atípico como el de mayor nivel educativo.

En cuanto al clúster de ingresos, se concentra en 27 municipios vecinos con bajos niveles, situados al sur, norte y oeste del Estado de 
México; el elevado I local de Moran para este fenómeno indica heterogeneidad de los datos $\mathrm{y}$, de forma contundente, que los bajos ingresos de estos municipios no están determinados de forma aleatoria, sino que dependen de la ubicación geográfica. Un dato preocupante, reflejo de la heterogeneidad del territorio, es el rango diferencial entre el municipio con menor ingreso (San José del Rincón, con $\$ 4,018.63$ ) y el del mayor (Metepec, con \$24,030.76): cinco veces el ingreso del más bajo.

La existencia de clústers bajo-alto, o alto-bajo, en el análisis del IDH, y sus componentes educación e ingresos, demuestran una divergencia muy marcada del desarrollo en esta entidad: por un lado, cuenta con un territorio rico y una población con alto grado de educación, salud e ingresos; y por otro, una región pobre y personas con bajos niveles de progreso, ingresos y educación. Tal parece que, a partir de la concentración poblacional en los dos grandes polos de atracción económica de este estado, Ciudad de México y Toluca, ha tenido lugar a una heterogeneidad en muchos de los fenómenos económicos y poblacionales, mientras ha dejado al descubierto la existencia de otras dos regiones con rasgos de desarrollo opuestos, donde se deben de realizar esfuerzos por buscar alternativas viables para mejorar las condiciones de vida.

Es pertinente seguir profundizando en el análisis de estos fenómenos particulares del desarrollo a fin de revertir las divergencias presentes en la entidad; futuras líneas de investigación podrían efectuar estudios confirmatorios basados en econometría espacial, con el propósito de establecer las causas de la heterogeneidad en el territorio.

\section{Bibliografía}

Acemoglu, Daron (2009), Introduction to modern economic growth. Princeton University Press, Estados Unidos.

Acevedo-Bohórquez, Ingrid y Velásquez-Ceballos, Emilson (2008), “Algunos conceptos de la econometría espacial y el análisis exploratorio de datos espaciales", en Ecos de Economia: A Latin American Journal of Applied Economics, Medellín.

Anselin, Luc (1988), Spatial econometrics: methods and models, Springer Science \& Business Media, Estados Unidos.

Anselin, Luc (1994), "Exploratory spatial data analysis and geographic information systems", en New Tools for Spatial Analysis, (17), Lisboa, pp. 45-54. 
Anselin, Luc (1995), "Local Indicators of Spatial Association-LISA", en Geographical Analysis, 27 (2), pp. 93-115.

Anselin, Luc y Bera, Anil K. (1998), "Spatial Dependence in Linear Regression Models with an Introduction to Spatial Econometrics", en Giles, David E.A. y Ullah, Aman (coord.), Handbook of Applied Economic Statistics, (237), Marcel Dekker, Nueva York.

Anselin, Luc; Syabri, Ibnu y Kho, Younginhn (2006), “An Introduction to Spatial Data Analysis", en Geographical Analysis, (38), Urbana, Illinois, $<5-22$, https://doi.org/10.1111/j.0016-7363.2005.00671.x> [16 de junio de 2018].

Aroca-González, Patricio (2000), “Econometría Espacial: Una herramienta para el análisis de la Economía Regional”, en IV Encuentro de la Red de Economía Social, Panamá.

Becerra-Lois, Francisco Ángel y Pino-Alonso, Jesús René (2005), “Evolución del concepto de desarrollo e implicaciones en el ámbito territorial: experiencia desde Cuba", en Economía, Sociedad y Territorio, (V), México, pp. 85-119.

Buzai, Gustavo D. y Baxendale, Claudia A. (2009), “Análisis exploratorio de datos espaciales", en Geografía y Sistemas de Información Geográfica (GEOSIG), (1977), Argentina, pp. 1-11.

Carrillo-Huerta, Mario Miguel (2001), "La teoría neoclásica de la convergencia y la realidad del desarrollo regional en México", en Problemas del Desarrollo, (32), IIEC-UNAM, México, pp. 107-133.

Chasco-Yrigoyen, Coro (2003a), "Econometría espacial aplicada a la predicción-extrapolación de datos microterritoriales", en Consejería de Economía e Innovación Tecnológica de la Comunidad de Madrid, Madrid.

Chasco-Yrigoyen, Coro (2003b), "Métodos gráficos del análisis exploratorio de datos espaciales", en Anales de Economía Aplicada, España, <http:// www.asepelt.org/ficheros/File/Anales/2003-Almeria/asepeltPDF/93. PDF $>$ [Mayo de 2018].

Chasco-Yrigoyen, Coro (2009), “Análisis exploratorio de datos espaciales al servicio del geomarketing”, en Instituto Lawrence R. Klein: Universidad Autónoma de Madrid, Madrid.

Conapo (Consejo Nacional de Población) (2013), Tasa de mortalidad infantil por municipio, 2010, México.

Gago, Alberto (1993), "Planificación y Desarrollo Regional”, en Curso Latinoamericano de Desertificación, Argentina.

García-Docampo, Manuel (2007), "El desarrollo local en el marco de los procesos de globalización”, en García Docampo, Manuel (coord.), Perspectivas teóricas en desarrollo local, Netbiblo, La Coruña, pp. 1 -33.

Goodchild, Michael F. (1987), “A spatial analytical perspective on geographical information systems", en International Journal of Geographical Information Systems, (1), pp. 327-334. 
Haining, Robert (1993), Spatial Data Analysis in the Social and Environmental Sciences, Cambridge University Press, Estados Unidos.

IGECEM (Instituto de Información e Investigación Geográfica, Estadística y Catastral del Estado de México) (2016), Producto Interno Bruto Municipal, 2015, Toluca.

Imco (Instituto Mexicano para la Competitividad) (2016), Índice de Competitividad Estatal, 2016, México.

Inafed (Instituto Nacional para el Federalismo y el Desarrollo Municipal) (2010), "Estado de México", Enciclopedia de los municipios y delegaciones de México, México.

INEGI (Instituto Nacional de Estadística y Geografía) (2010), Censo de población y vivienda 2010. Cuestionario ampliado: Características de las personas, México <http://www.inegi.org.mx/est/contenidos/proyectos/ ccpv/cpv2010/Default.aspx $>$ [Junio de 2018].

INEGI (2015a). Encuesta Intercensal 2015, México, <http://www.beta.inegi. org.mx/proyectos/enchogares/especiales/intercensal/> [Junio de 2018].

INEGI (2015b), PIB y cuentas nacionales. México, <http://www.inegi.org.mx/ est/contenidos/proyectos/cn/pibe/> [Junio de 2018].

INEGI (2015c), Información por entidad: Estado de México, México, $<$ http://cuentame.inegi.org.mx/monografias/informacion/mex/default. aspx?tema $=$ me\&e $=15>$ [Junio de 2018].

INEGI (2017), PIB por entidad federativa, anual, México <http://www.inegi. org.mx/est/contenidos/proyectos/cn/pibe/tabulados.aspx $>[20$ de junio de 2018].

Meier, Gerald M. y Stiglitz, Joseph E. (2002), Fronteras de la economía del desarrollo. A.C.S.A., Washington, D.C.

Moran, Patrick Alfred Pierce (1950), "Notes on Continuous Stochastic Phenomena", en Biometrika, (37), Oxford, pp. 17-23.

Moreno-Serrano, Rosina y Vayá-Valcarse, Esther (2000), Técnicas econométricas para el tratamiento de datos espaciales: la econometría espacial. Universitat de Barcelona, Barcelona.

Mozas-Moral, Adoración y Bernal-Jurado, Enrique (2006), “Desarrollo territorial y economía social”, en Revista de Economía Pública, Social y Cooperativa, (55), España.

PNUD (Programa de Naciones Unidas para el Desarrollo) (1990). Human Development Report 1990, ONU Press, Nueva York.

PNUD (2014), Índice de Desarrollo Humano Municipal en México (2010): nueva metodología, Sonideas, Ciudad de México.

Sen, Amartya (1990), "Development as capability expansion", en Griffin, Keith y Knight, J. (coords.), Human Development and the International Development Strategy for the 1990s, Londres.

Sen, Amartya (1996), "Capacidad y bienestar", en Nusbaum, Martha C. y Sen, Amartya, La calidad de vida, Fondo de Cultura Económica, México. 
Sen, Amartya (2001a), Development as freedom, Oxford University Press, Oxford.

Sen, Amartya (2001b), "Las teorías del desarrollo en el siglo XXI", en Leviatán: Revista de Hechos e Ideas, (84), pp. 65-84.

Todaro, Michael (1982), Economía para un mundo en desarrollo: Introducción a los principios problemas y políticas para el desarrollo, Fondo de Cultura Económica, México.

Todaro, Michael (1988), Desarrollo económico del tercer mundo. Alianza Universidad, Madrid. 